\title{
Based on the Complete CDA Standard Clinical Document Editor Research and Development
}

\author{
Jun He, Liang Xiao \\ School of Computer Science, Hubei University of Technology, Wuhan, China. \\ Email: hejun.0819@gmail.com, lx@mail.hbut.edu.cn
}

Received 2013

\begin{abstract}
The design and development of a kind of fully comply with an HL7 standard clinical CDA document editor for generating standard CDA standard XML file, and can extract the other clinical commercial software generating clinical document XML- related content, and modified into standard CDA XML documents, used for data exchange, data mining and clinical decision support.
\end{abstract}

Keywords: The HL7; The HL7 CDA; Clinical Document

\section{Introduction}

Because the traditional paper clinical documents (including course records, discharge summary, etc.) have the problems in the writing norms, preservation, resource sharing, as well as the high speed development of informatization for medical institutions to bring fast, safe, advanced management, clinical application, electronic clinical document arises at the historic moment. Electronic clinical document first purpose is to resource standard and safety preservation, but as the electronic clinical document data is unceasingly rich, and the clinical staff, and the researchers of potential rich clinical data in the clinical research of great value, in urgent need of these rich data to exchange and share. But many domestics and international relevant software developers in the beginning of software architecture did not form a unified and standard data exchange standard, make clinical data transmission and exchange exist some difficulties.

The HL7 established conveniently. The HL7 is a after the American national standards institute approved standards development organizations, it has made a number of information exchange protocols, and get international recognition and extensive practice. It has gathered through different manufacturers, medical institutions, organizations, establishing of the standardization of the health information transmission protocol, make each hospital in heterogeneous system can make data interaction. And CDA (Clinical Document Architecture) as the HL7 standard set, as a part of the clinical document in exchange for the purpose is to describe the clinical document structure and semantic document marking standard $^{1}$. It details the clinical document structure and semantic, is also a clear, complete object, can contain text, images, audio and other multimedia content. But, so far, both at home and abroad such as electronic medical records related to the clinical document design exists some insufficient and defect:

1) Do not completely comply with the HL7 standard, especially in the design of electronic medical records, do not completely comply with the standard of CDA, generate XML file cannot be through the HL7 CDA syntax and semantic the authentication, in the isomerism cases, clinical data exchange and sharing idea is just stay on paper.

2) No software can extract other clinical software developers generating XML document, so can't for related data extraction, analysis and higher level of utility

This research mainly include 1) design and develop fully comply with the HL7 CDA standard based on web clinical document editor, and can extract the other software developers generating XML documents 2) extracting clinical expert knowledge and clinical document content and changed into computer can identify language, form a maintainable clinical knowledge base 3) in this clinical knowledge base, when the clinical medical staff use the program to make order, write disease, the system will give the clinical guidance and the decision support, and finally form a maintainable, knowledge base is unceasingly rich medical decision support system. This paper firstly introduced the HL7 related content, then detailed illustrate the CDA technical details, and the third part, we will describe development and function display of the clinical document editor base on Web, finally we 
will summarize our research work, and introduce the follow-up research direction and contents.

\section{Project Overview}

Clinical decision support systems (CDSSs) have been hailed for their potential to reduce medical errors and increase health care quality and efficiency. At the same time, evidence-based medicine has been widely promoted as a means of improving clinical outcomes, where evidence-based medicine refers to the practice of medicine based on the best available scientific evidence. The use of CDSSs to facilitate evidence-based medicine therefore promises to substantially improve health care quality [1]. The project mainly through the establishment of clinical knowledge base for clinical decision support provides a strong background support, and overall vision as shown in Figure 1: design a clinical document editor for clinical staff to complete the document editing, and in full compliance with $t$ the CDA standard of XML document on the one hand, on the other hand extracts XML format generated by other software vendors clinical specify the content of the document, and save it as a legitimate CDA document; through more than two parts of the data acquisition and the use of natural language processing, data mining technology, the formation of new clinical guidelines.(Extract data for a variety of diseases, and also make a number of clinical guidelines); Transform the existing clinical guidelines, and own clinical guidelines to clinical data that the computer can be

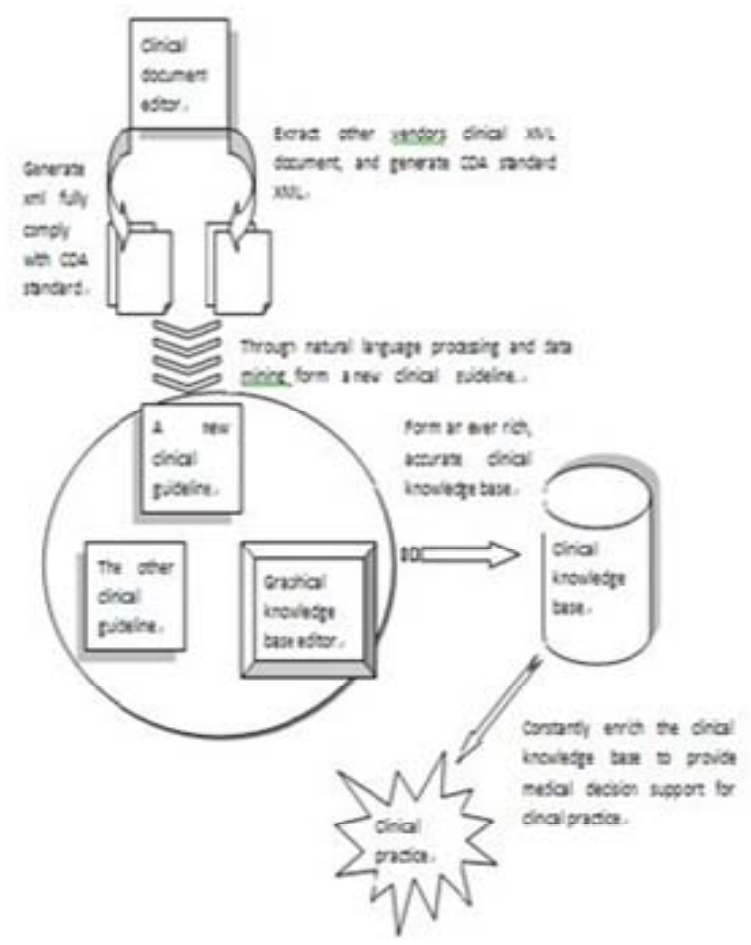

Figure 1. identified, as well as develop a graphical tool for clinical experts to edit and maintain clinical expertise knowledge, and ultimately form a continuously maintainable ,data continuously enriched ,and accurate clinical knowledge base to provide accurate, efficient clinical decision support for a variety of clinical practice.

\section{Introduction of HL7}

Health Level Seven organization was founded in 1987 by dr. SamSchultz in Pennsylvania university hospital hosted a meeting contributed to an HL7 organization and communication standard was born. With many users, manufacturers, consultant organization to join, the HL7 team is growing, and established the HL7 working group finally. Health information exchange standards in HL7 (Health Level 7) is a health information transfer protocol, standardized electronic transfer agreement between the different applications in the medical field. A collection of different vendors HL7 standard format used to interface between the design of application software, which will allow data interaction between heterogeneous systems, various medical institutions.HL7's main application areas is HIS / RIS, communication between the specification HIS / RIS system and its equipment, it is involved in all aspects of the wards and patient information management, laboratory, pharmacy, radiology systems, toll collection system. HL7's aim is the development of the hospital data transfer protocols and standards, to standardize clinical medicine and management information format, reducing the cost of the hospital information system interconnection, to improve the level of information sharing of data between the hospital information system. Health Level 7 Level 7 "is the highest layer in the OSI seven-layer model, the seventh floor. But this is not to say that it follows the seventh layer of the OSI definition of data elements, it is only used to constitute its own abstract data types and encoding rules. It also does not require the specification of how to support the first to sixth layers of the OSI data. As standard, the HL7's goal is:

1) HL7 standard should support the exchange of data in different technological environments, should also support a variety of programming languages and operating systems, as well as support a variety of communications environments.

2) Support both single stream and multiple data stream two means of communication.

3) The maximum compatibility, reserved special table, encoding defined for different users, and news segments (such as: HL7's Z-segments).

4) The standards must have the scalability to support new requirements, which include the extension of the agreement itself and is compatible with the existing system and the new system.

5) The standards should be in full reference to existing 
communication protocols based on widely accepted industry standard.

6) HL7 long-term goal is to develop a method for medical institutions, electronic data interchange standards or protocols.

\section{HL7 CDA Technology}

Clinical documentation is a completely object, including text, images, sound and other multimedia content, it has sustained, operability, can be identified, integrity, readability. CDA have the following characteristics [2]: 1) marked the CDA with XML language. 2) CDA inherit the data type of the first edition of HL7RIM3 and HL7 3 release.3) complete CDA will include a set of hierarchical document specifications, this level formed structure of the document."

A CDA document by document type definition, composition head (CDA Header), body (CDA Body). Document Type Definition norms and constraints of the document, and the way to use the CDA version is the DTD that the document type definition; the CDA Header determine the classification of the document, provide the identification information, respondents data, patient information, provider information; CDA Body is composed of a nested set, a total of four content sets: section, paragraph, lists, tables. The content sets contain content and optional title, including plain text, links or multimedia data. CDA Header and the CDA Body are defined using the HL7 RIM data type [3].

\section{Clinical Editor Development and Function Illustrate}

The document editor using JSP development, and using DOM (Document Object Model) to process and read the XML documents. JSP development is conducive to distributed deployment; DOM parsing an XML document mechanism biggest feature is that the entire XML data as a one-time loaded into system memory object parsed into a tree, and can make use of the parent-child relationship between the mark throughout XML data structure on the high-speed browsing. Editor function is mainly divided into two blocks: using the editor to save the editor's content into fully compliance with the CDA standard of XML document and can pass the official CDA's validator ; reading XML documents generated by other vendors, to extract critical information to for clinical guide data studies; and save it as compliant XML documents, Figure 2 is the clinical document design diagram, Figure 3 is illustrating classes and functions:

\section{Summary}

Clinical document editor design complete marks the whole project studies have been done to the first part,

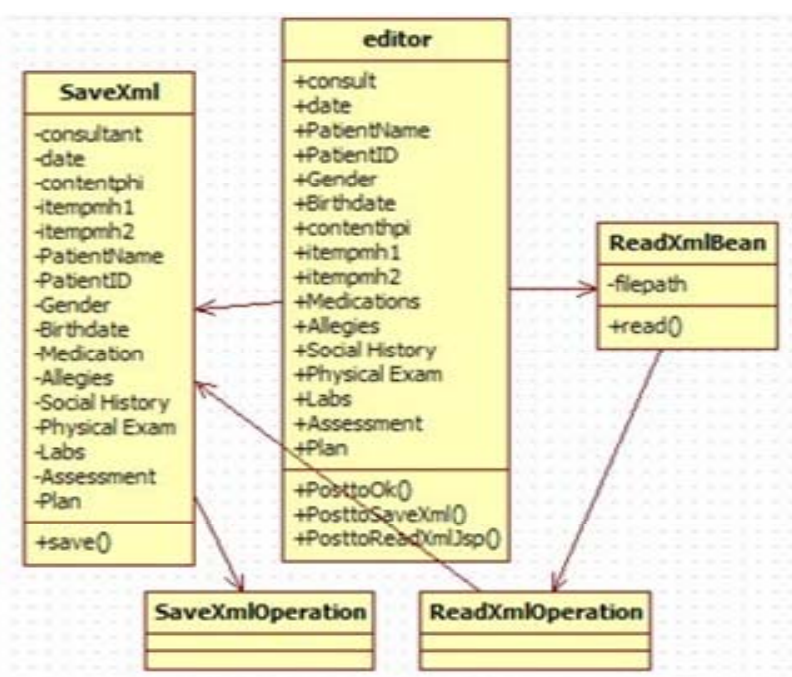

Figure 2.

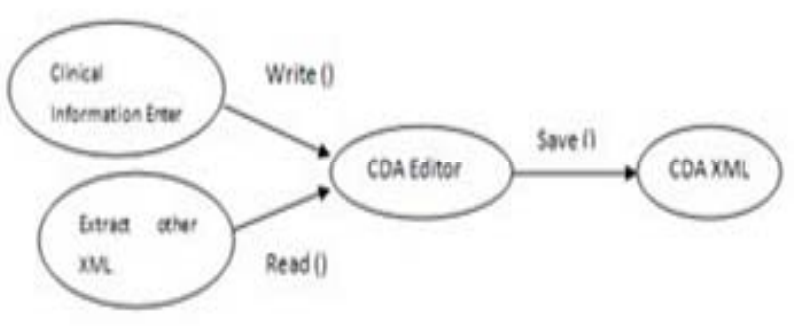

Figure 3.

Good Health Clinic Consultation Note

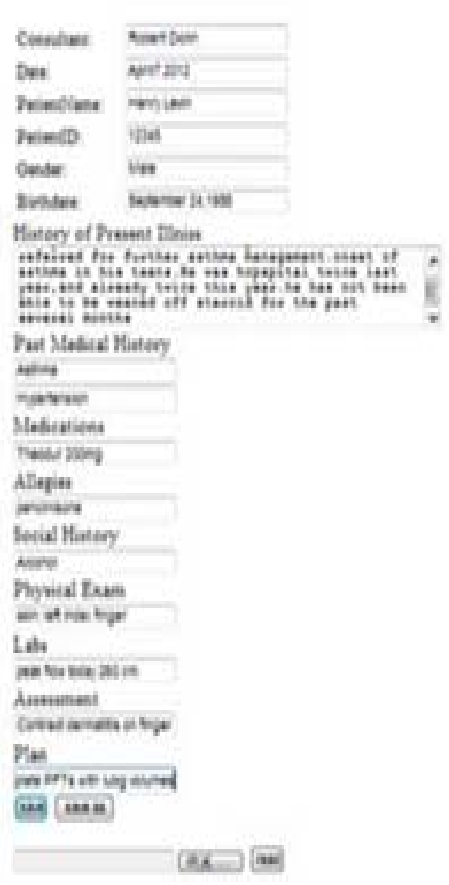

Figure 4. 


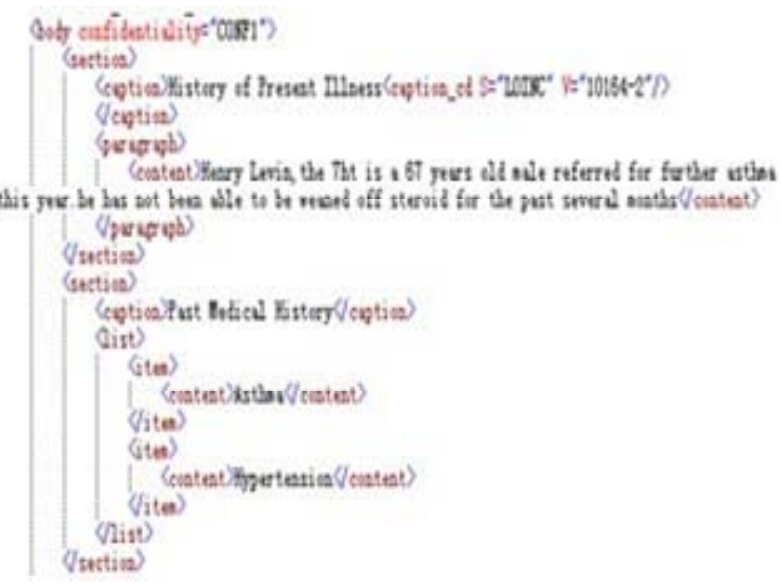

Figure 5.

next we will set the editor into specific clinical practice, further improve the clinical document editor function and technical details, make its are on the function more rich, more flexible architecture, and using it to extract other software company generate XML document, extraction rich, precise and effective use of clinical data, for the development of new clinical guidelines lay a solid data support. Guidelines are not integrated into an electronic patient record [4,5]. In a follow-up research work, clinical guidelines for the structural expression and clinical practice of decision support would be our research focus.

\section{Acknowledgment}

This work is supported by National Natural Science Foundation of China under Grant Number 61151001.

\section{REFERENCES}

[1] Kensaku Kawamoto, Caitlin A Houlihan "Improving clinical practice using clinical decision support systems" 2005,PP.1-3

[2] Robert H. Dolin, MD, Liora alschuler, “The HL7 Clinical Document Architecture”, 5/23/2001,PP.1-10

[3] M.Eichelberg,T.Aden,”Electronic health record standards-a brief overview”,2006,PP.1-6

[4] Xiao L., Cousins G., Courtney B., Hederman L., Fahey T., Dimitrov B.D., "Developing an Electronic Health Record (EHR) for Methadone Treatment Recording and Decision Support”, BMC Medical Informatics and Decision Making 11:5, 2011.

[5] Xiao, L., Cousins, G., Fahey, T., Dimitrov, B., Hederman, L., "Developing a rule-driven clinical decision support system with an extensive and adaptive architecture”, Proceedings of the 14th International Conference on E-health Networking, Application \& Services (HealthCom'2012), pp.250-254, 2012. 